\title{
CAPEX study for grid dependent multi-layer IP/MPLS-over-EON using relative BV-WSS costs
}

\author{
O. Pedrola ${ }^{1}$, L. Velasco ${ }^{1 *}$, A. Castro ${ }^{1}$, J. Fernández-Palacios ${ }^{2}$, D. Careglio ${ }^{1}$, G. Junyent ${ }^{1}$ \\ ${ }^{1}$ Universitat Politècnica de Catalunya (UPC), Barcelona, Spain \\ ${ }^{2}$ Telefónica I+D, Madrid, Spain \\ *e-mail: lvelasco@ac.upc.edu
}

\begin{abstract}
The impact of the frequency grid on the CAPEX required to deploy a multi-layer IP/MPLS-over-EON is analyzed. Results showing significant savings at the electronic layer motivate the introduction of narrower grids with enhanced BV-WSS.

(C) 2011 Optical Society of America

OCIS codes: (060.0060) Fiber optics and optical communications; (060.4250) Networks
\end{abstract}

\section{Introduction}

The emergence of new disruptive bandwidth intensive services and applications has led to a huge surge of IP traffic which, ultimately, has brought to light the clear granularity mismatch between the client layer and current wavelength-routed optical layer. This issue results in a highly inefficient use of the network capacity, and consequently, in multi-layer networks requiring a large amount of highly expensive, power-consuming IP/MPLS equipment to be installed for grooming/aggregation purposes. In this context, Elastic Optical Networks (EON) [1, 2], which provide a highly flexible, spectrally efficient use of resources, have emerged as a potential candidate for next-generation optical transport networks. EONs, which leverage upon key advances in optical multi-level modulation techniques and the design of both bandwidth-variable transponders (BV-Ts) and bandwidth-variable wavelength selective switches (BV-WSSs), are regarded as a viable alternative to the not yet mature optical packet switching (OPS) technology enabling both sub- and super-wavelength traffic accommodation.

In EONs, the available optical spectrum is divided into a set of frequency slots (FSs) of a fixed (finer) spectral width, e.g. $25 \mathrm{GHz}, 12.5 \mathrm{GHz}$ or even $6.25 \mathrm{GHz}$, in comparison to the current ITU-T DWDM rigid frequency grid $(50 \mathrm{GHz})$ [3]. The number of contiguous FSs an optical demand occupies depends on the requested bit-rate, the modulation technique and the frequency grid [2]. Thus, such narrower grids allow for efficient spectrum utilization and favor grooming data directly at the optical layer instead of requiring costly IP/MPLS equipment for such functionality. Since network CAPEX is a figure network operators are always striving to reduce, the introduction of EON technology is of paramount importance for future multi-layer networks. However, while reducing the need for grooming at the IP/MPLS layer, this more advanced optical technology will also imply higher costs at the optical layer given the highly demanding (grid-dependent) filtering characteristics required in each BV-WSS.

In this work, we study the effect of the different frequency grids on the CAPEX required to deploy a multilayer IP/MPLS-over-EON network architecture under a variety of traffic profiles. Then, we consider a relative cost for BV-WSSs so as to determine which frequency grid will better address network operator's needs for costeffective, spectrum-efficient network architectures.

\section{BV-WSS Cost Computation for Multilayer IP/MPLS-over-EON Design}

The following problem is solved to find the cost of the BV-WSS.

Given: (1) a network topology represented by a graph $G_{o}(N, L)$, being $N$ the set of locations and $\mathrm{L}$ the set of fiber links connecting two locations; (2) a set $S$ of available slots of a given spectral width for each link in $L$; (3) the virtual network represented by a graph $G_{V}\left(N_{V}, E\right)$, being $N_{V}$ the subset of locations where IP/MPLS nodes can be placed and $E$ the set of virtual links defining the connectivity among those IP/MPLS locations; (4) a set $D$ of IP/MPLS demands to be transported; (5) IP/MPLS equipment cost, specified by a fixed cost for every type of IP/MPLS node and BV-T port; (6) optical nodes cost, which include a fixed cost for common hardware, and a variable cost which depends on the nodal degree and the cost of the BV-WSSs (see [1]); (7) a cost for every optical amplifier (OA) to be equipped in the used fiber links and a cost per $\mathrm{km}$ and $\mathrm{GHz}\left(c_{f_{0}}\right)$ of using the already deployed fiber;.

Output: (1) the optical network, including patch panels, optical nodes with the number of optical interfaces and fibers; (2) the configuration of IP/MPLS nodes in terms of capacity and number and bit-rate of BV-Ts.

Objective: Minimize the expected CAPEX for the network designed for the given set of demands.

The research leading to these results has received funding from the European Community's Seventh Framework Programme FP7/2007-2013 under grant agreement $n^{\circ} 247674$ STRONGEST project. Moreover, it was supported by the Spanish science ministry under the FPU program and the TEC2010-18522 DOMINO project. 
To find the cost for each grid-dependent BV-WSS, we design the IP/MPLS-over-EON for each frequency grid with the aim of minimizing the CAPEX. To this end, we first fix different costs for the BV-WSSs for each frequency grid; then, we graphically estimate the cost for the BV-WSSs operating in different grids by choosing a reference $50 \mathrm{GHz}$ grid BV-WSS cost, which provides us with the maximum allowable CAPEX investment for every grid, and consequently, the maximum cost for the different BV-WSSs.

The BV-WSS Cost Computation problem was modeled by means of an integer linear programming (ILP) formulation based on [4]. However, its exact solution becomes impractical when real-sized network and traffic instances are considered. For this reason, we developed a greedy randomized adaptive search procedure (GRASP) construction algorithm based on the sample greedy approach [5]. Considering very small problem instances we compared the performance of both the ILP and heuristic methods and found that the GRASP-based heuristic is able to provide a much better trade-off between optimality and complexity.

\section{Illustrative numerical results}

In our experiments, we execute the GRASP-based heuristic considering the 21-node Spanish Telefónica network (see Fig. 1) and the three different traffic profiles (TP) reported in Table 1. The average amount of Tb/s offered to the network is equal for all TPs. Demand bit-rates are 10, 40, 100 or 400Gb/s. For the sake of a comprehensive study, TPs range from a scenario with a high number of lightly loaded demands (only 24.1Gb/s on average in TP-1) to a scenario with fewer demands but with a higher bit-rate (80Gb/s on average in TP-3). These TPs represent the evolution in the expected bandwidth necessities for the years to come.

We assume $N_{V}=N$ and that only those locations in blue in Fig. 1 can be source or destination of IP/MPLS demands. The remaining locations can be either an empty location if no demand traverses such location, a bandwidth-variable wavelength cross-connect (BV-WXC) with or without IP/MPLS equipment, or a patchpanel connecting optical fibers if neither IP/MPLS nor BV-WXC functionality is needed.

In Table 2, the network equipment costs in cost units (c.u.) and features used to compute the CAPEX are provided. IP/MPLS nodes and BV-Ts features have all been obtained from discussions currently being held within the STRONGEST project [6]. Since the cost for the different BV-WSS (i.e., the finer the grid the higher the cost) is still unknown, we make use of relative values to estimate, given the target CAPEX, their maximum affordable cost. For the data transmission, we assume QPSK as the modulation format, and hence, a factor of 2 is used to compute the number of FSs each demand requires [2]. In order to route the demands, a set of $k$ shortest paths is pre-computed over both the physical and virtual network topology. Finally, we considered an optical spectrum width of $2 \mathrm{THz}$.

Fig. 2 shows, for each frequency grid and for a set of relative cost values for the BV-WSS, the network CAPEX, the total number of BV-Ts installed, and the average bit-rate per BV-T in the network constructed. Each of the points in the plots corresponds to an average over 10 independent runs of the heuristic algorithm. For each TP, we consider both a highly loaded scenario (4.5Tb/s are injected into the network) and a medium one $(3.5 \mathrm{~Tb} / \mathrm{s})$.

As illustrated, the effectiveness of narrower grids in grooming data directly at the optical layer, thus reducing network CAPEX, is clearly dependent on the TP considered. Whilst in TP-1 the introduction of finer grids allows for the spectrum to be better exploited, and thus to achieve further benefit, in TP-2 the 6.25GHz grid provides the same performance as the $12.5 \mathrm{GHz}$, and eventually in TP-3 the main improvement is obtained just by considering a $25 \mathrm{GHz}$ grid. Similarly, as shown in the rest of the plots, the number of BV-Ts installed and their average bit-rate are also a consequence of both the frequency grid and TP profiles evaluated. The use of finer frequency grids entails a higher number of BV-Ts but with a considerably lower average bit-rate per BV-T.

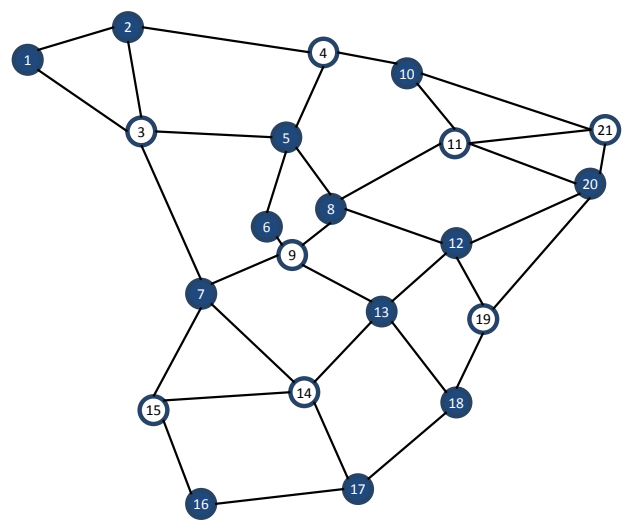

Fig. 1. The 21-node Spanish Telefónica network topology.
Table 1. Traffic profiles considered

\begin{tabular}{|c|c|c|c|}
\hline Traffic Profile & TP-1 & TP-2 & TP-3 \\
\hline Avg. demand bit-rate (Gb/s) & 24.1 & 52.0 & 80.0 \\
\hline
\end{tabular}

Table 2. Network equipment cost. IP/MPLS nodes, BV-Ts and OAs.

\begin{tabular}{|l|c|c|c|c|c|}
\hline $\begin{array}{c}\text { IP/MPLS } \\
\text { Node }\end{array}$ & Class 1 & Class 2 & Class 3 & Class 4 & Class 5 \\
\hline $\begin{array}{l}\text { Capacity } \\
\text { (Gb/s) }\end{array}$ & 160 & 320 & 640 & 1280 & 2560 \\
\hline Max. ports & 4 & 8 & 16 & 32 & 64 \\
\hline Cost (c.u.) & 9 & 13.5 & 19.5 & 67.5 & 150.57 \\
\hline
\end{tabular}

\begin{tabular}{|l|c|c|c|c|}
\hline \multicolumn{1}{|c|}{ BV-T } & $\mathbf{1 0 ~ G b / s}$ & $\mathbf{4 0 ~ G b} / \mathbf{s}$ & $\mathbf{1 0 0} \mathbf{~ G b} / \mathbf{s}$ & $\mathbf{4 0 0} \mathbf{~ G b} / \mathbf{s}$ \\
\hline Max. reach $(\mathrm{Km})$ & 2500 & 2000 & 1000 & 400 \\
\hline Cost (c.u.) & 2.5 & 7.625 & 20.625 & 65.625 \\
\hline
\end{tabular}

\begin{tabular}{|c|c|}
\hline Optical Amplifiers (OA) (c.u.) & 5 \\
\hline cfo (per GHz and Km) & 0.02 \\
\hline
\end{tabular}


a)
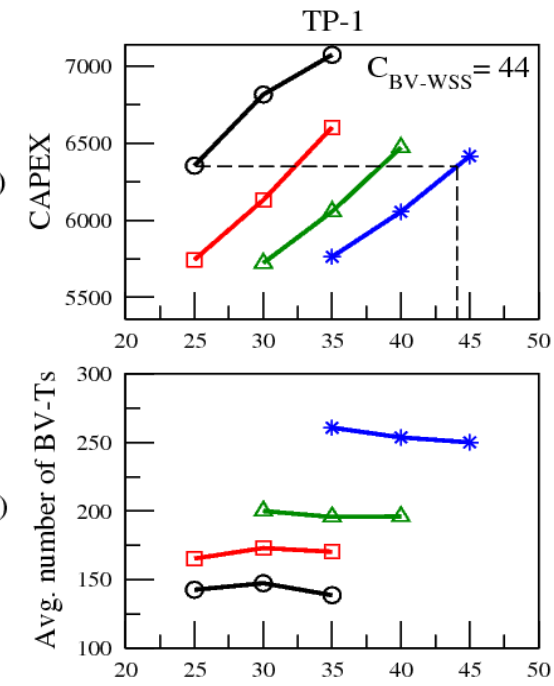

c)

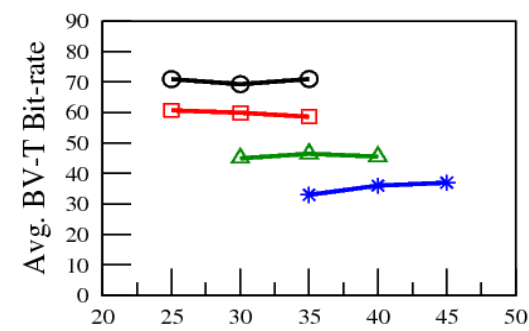

TP-2
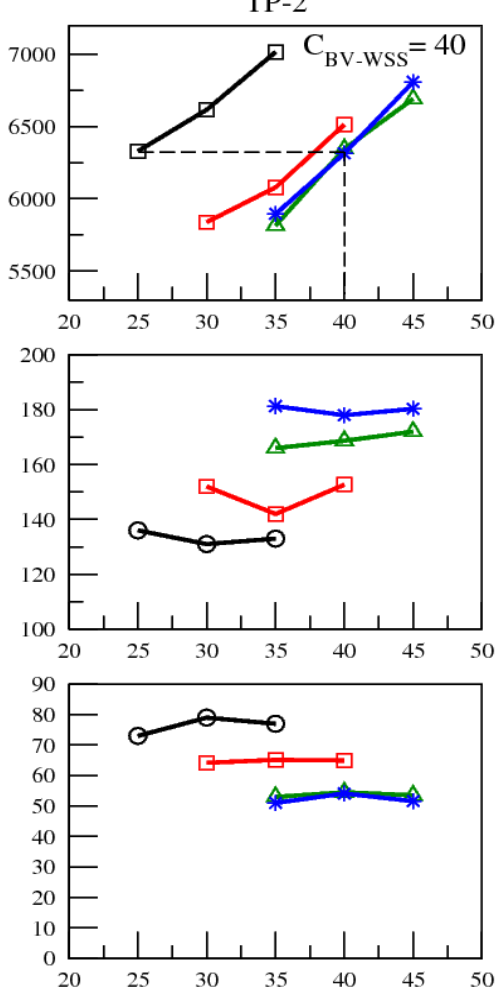

TP-3
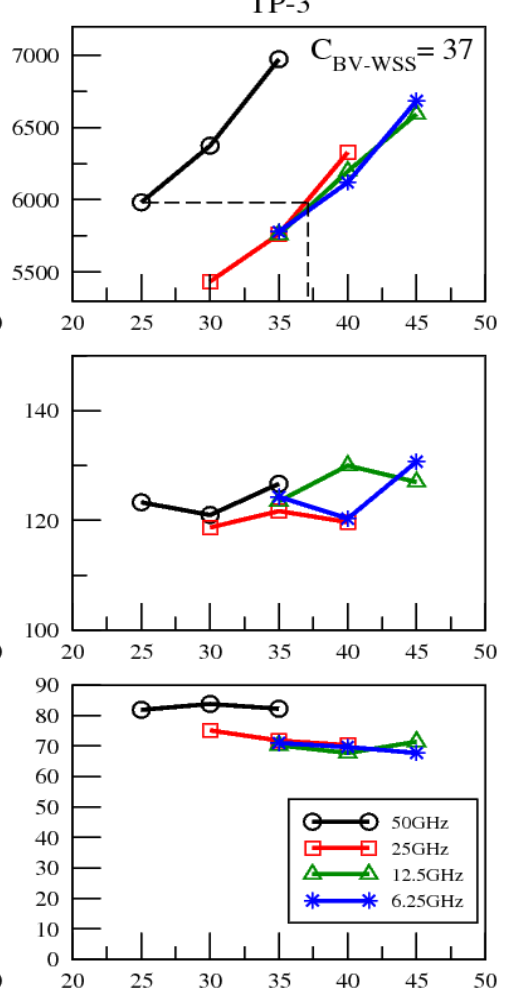

Fig. 2. a) Network CAPEX (IP/MPLS and optical equipment cost), b) average number of BV-Ts installed and c) average bit-rate per BV-T as a function of the relative cost of one BV-WSS. The four different optical grids are considered.

In order to estimate the maximum cost increment for the BV-WSS, we use as benchmark reference a BVWSS cost of 25 units for the $50 \mathrm{GHz}$ grid. As shown in Fig. 2, for the traffic profile with lighter demands (TP-1), the cost of the BV-WSSs can be, for the same network CAPEX, as high as 44 c.u., that is, $76 \%$ more expensive than the one used in the $50 \mathrm{GHz}$ grid. However, when the on-average bit-rate of the demands increases in TP-2 and TP-3, the cost of a BV-WSS decreases to about 40 c.u. (60\%) and 37 c.u. (48\%), respectively.

From a temporal perspective given by the on-average bit-rate of demands, high cost increments can be assumed for a $6.25 \mathrm{GHz}$ grid BV-WSS in the near future. However, these investments will not be profitable considering the expected traffic evolution. On the contrary, investments in EON using the $12.5 \mathrm{GHz}$ or even the $25 \mathrm{GHz}$ grid seem cheaper in the short term and more appropriated for long term scenarios.

\section{Conclusions}

In this paper, we have evaluated the impact of several frequency grids on the cost of BV-WSSs, the key component of a BV-WXC, in the multi-layer IP/MPLS-over-EON network design problem. Such problem has been first formally modeled by means of an ILP formulation and then solved using a GRASP-based metaheuristic algorithm considering real-sized network and traffic instances.

Results show that the performance of the different frequency grids is strongly dependent on the TP considered. We observed that the reduction in costly IP/MPLS equipment achieved by using narrower grids allows for substantial increments in the cost of BV-WSSs.

\section{References}

1. M. Jinno et al., "Spectrum-efficient and scalable elastic optical path network: architecture, benefits, and enabling technologies," IEEE Commun Mag., vol. 47, pp. 66-73, 2009.

2. M. Jinno et al., "Distance-adaptive spectrum resource allocation in spectrum-sliced elastic optical path network," IEEE Commun. Mag., vol. 48, pp. 138-145, 2010.

3. ITU-T G.694.1, “Spectral grids for WDM applications: DWDM frequency grid,” May 2002.

4. L. Velasco, M. Klinkowski, and M. Ruiz, "Modeling the Routing and Spectrum Allocation Problem for Future Elastic Optical Networks,” Technical Report UPC-DAC-RR-CBA-2011-8, 2011.

5. M. Resende and C.C. Ribeiro, “GRASP: Greedy Randomized Adaptive Search Procedures,” Search Methodologies, Springer, 2011.

6. "Scalable, Tunable and Resilient Optical Networks Guaranteeing Extremely-high Speed Transport (STRONGEST)" project: http://www.ict-strongest.eu/. 